\title{
Re: Outcomes of Microdissection Testicular Extraction in Men with Nonobstructive Azoospermia due to Maturation Arrest
}

\author{
Bernie AM1, Shah K1, Halpern JA1, Scovell J2, Ramasamy R2, Robinson B33, Schlegel PN4
}

1 Weill Cornell Medical College, Clinic of Urology, New York, USA

2Baylor College of Medicine, Houston, Texas, USA

3 Weill Cornell Medical College, Clinic of Pathology and Laboratory Medicine, New York, USA

4 Weill Cornell Medical College, Clinic of Urology, New York, USA

Fertil Steril 2015;104:569-573.e1. doi: 10.1016/j.fertnstert.2015.05.037. Epub 2015 Jun 18.

\section{EDITORIAL COMMENT}

Previous studies showed that, compared with men with late maturation arrest (MA), men diagnosed with early MA had a decreased sperm recovery rates by testicular sperm extraction (TESE). In this study, the authors classified patients with non-obstructive azoospermia (NOA) as either early or late, and focal or diffuse and compare sperm recovery rates by a single surgeon using micro TESE operation technique. Early MA was defined in patients whose pathology results showed spermatogenetic arrest at the spermatogonia or spermatocyte stage; and late MA in those arrest occurred at spermatid stage. On biopsy, MA may also vary in its heterogeneity from focal to diffuse pattern in whole seminiferous tubule of the pathology sample. The authors concluded that men with late and focal MA have a higher sperm recovery rate as compared to men with early and diffuse MA. In the presence of MA pathology, a high level of follicle-stimulating hormone (FSH) may predict heterogeneity of testicular tissue. The authors advise the clinicians to be aware of those men with normal FSH and normal testicular volume, with NOA, may have particularly poor sperm recovery rate.

Emre Bakırcıoğlu, MD

\section{Re: Fourteen Babies Born after Round Spermatid Injection into Human Oocytes}

\author{
Tanaka A1, Nagayoshi M1, Takemoto Y1, Tanaka I1, Kusunoki H2, Watanabe S3, Kuroda K4, Takeda S4, \\ Ito $\mathrm{M} 5$, Yanagimachi $\mathrm{R} 6$
}

IInstitute for ART, Clinic of Saint Mother Obstetrics and Gynecology, Fukuoka, Japan

2Kobe University, Graduate Faculty of Agriculture, Faunal Diversity Sciences, Kobe, Japan

3 Hirosaki University Graduate Faculty of Medicine, Department of Anatomical Science, Hirosaki, Japan

4Juntendo University Faculty of Medicine, Department of Obstetrics and Gynecology, Tokyo, Japan

5 Hamamatsu University Faculty of Medicine, Department of Infectious Diseases, Hamamatsu, Japan

6University of Hawaii Medical Faculty, Department of Anatomy, Physiology, and Biochemistry, Honolulu, USA

Proc Natl Acad Sci USA 201524;112:14629-34. doi: 10.1073/pnas.1517466112. Epub 2015 Nov 2.

\section{EDITORIAL COMMENT}

In some infertile men, who have nonobstructive azoospermia, round spermatids (haploid male germ cells that have completed meiosis) are the most mature cells that are found after testicular sperm extraction operation. The microinjection of a round spermatid into an oocyte is called round spermatid injection (ROSI). Currently, human ROSI is considered a very inefficient procedure and of no clinical application. In this study, the authors reported the birth and development of 14 children born to twelve women following ROSI of 734 oocytes activated by an electric current. They concluded that the success was the ability to identify round spermatids accurately and oocyte activation by electric stimulation. All children were born after ROSI without any unusual physical, mental, or epigenetic problems. In this recent study, it has unexpectedly been shown that round cells, which are immature spermatozoa, may have ability to achieve pregnancy and live birth in human. ROSI may be considered an option and last chance for men whose germ cells are unable to develop beyond the round spermatid stage to have their own genetic offspring. 